\title{
Analysis of Choice and Application of Costume Color based on the Perspective of Character Image Design
}

\author{
Xia Xuemin \\ Jiangsu Open University, Nanjing 210036, Jiangsu, China
}

Keywords: Character image; Costume color; Shape; Age; Overall

\begin{abstract}
The global features of character image design determines that all the design elements should be grasped, thereinto, as an important part of the design, costume color choice has the key effect and core value, and directly affects the overall effect of character image design, therefore, it is of vital significance to study the choice and application of costume color in character image design. In this thesis, on the basis of the understanding of the important role of costume color, as well as the practices of character image design, the specific methods for choice and application of costume color were summarized and concluded in this thesis, which is of certain reference value.
\end{abstract}

\section{Introduction}

Character image is the comprehensive embodiment of individuals' internal accomplishment and external form. With the improvement of the overall material standard of living in the society, people are pay increasing attention to and demand more on image design. Besides, the integration of image design into cosmetics, clothing and other industries has led to rapid development of image design in recent years. At present, character image design has entered the stage of life design, namely, paying close attention to people's thinking and behaviors in a people-oriented mode and with the purpose of creating new lifestyle and adapting to personality. This determines that character image design has been gradually transformed into a comprehensive and practical art which integrates creativity, modeling, cosmetology, make-up, clothing and many other subjects into one. This kind of art is aimed at shaping and creating proper and perfect image that conforms to the occupation, disposition, age and culture of the characters by means of formative arts and through make-up design, hair style design and costume design. It can be seen that character image design in nowadays society is a complex and comprehensive process, and every link of the design needs to be studied thoroughly, so as to achieve the internal and external unity of character image design. In this thesis, the perspective of costume color is mainly adopted to expound its importance in the process of character image design, as well as the specific design principles and methods.

\section{The role of costume color in character image design}

Chanel, the big fish in the fashion circle, made a very straightforward exposition of the important position of costume in character image: "When your wearing is unassailable, people will notice you." [1]Costume is like a reflective mirror, which can reflect the customs and features of different countries as well as the cultural characteristics of different eras, and display the temperament and look of different characters. Costume has developed from the initial "for warming, no patterns" to "bright and colorful", and interlaced with other colorful things created by humans, thus constituting the indispensable color art in human life. Costume is made up of three major factors of color, shape and material, however, color is the first thing that jumps into viewers' sight, followed by the style and pattern, and finally the material and craft. Apparently, among the numerous factors, color factor is vivid and eye-catching, and occupies a decisive position. Color can be the first to arouse people's attention, and leaves a deep impression on people, which determines the important role of it in the design of character image. 


\section{The aesthetic appreciation role of costume color}

Color is the life and soul of costume. Decent costume and appealing colors can not only bring people a delectable feeling, but also give confidence and joy to the wearer. Meanwhile, color can bring people vast imaginary space. For example, the pretty and charming maid dressed in skirt with the hemline swaying in the wind and with natural and fresh streamline patterns can remind people of pink flowers and, romantic and elegant spring; the tall and sexy model dressed in a blue evening dress can remind people of the blue vast sky and the sea; the pinkish purple tippet and the grey purple long dress can remind people of the joy when seeing flowers blooming.

\section{The artistic creation role of costume color}

\section{Color and fabric material}

The creation of aesthetic feeling of costume color cannot be separated from costume material, various costume colors are tied to fabric. After the costumes made from different fabrics and with different colors are worn on the human body, the costume colors are immediately transformed from plane form into three-dimensional form. Therefore, in the selection and application of costume color, the masters not only take into consideration the plane effect of colors, but also pay more attention to the 3D effect, so that the costume can produce visual balance in every angle after being worn, furthermore, the masters even never neglect the color processing on both sides and the back. The perfect presentation of gorgeous colors in different fabrics and materials is as follows. Figure 1 shows the costume made from feathers, and Figure 2 shows the costume made from paper.

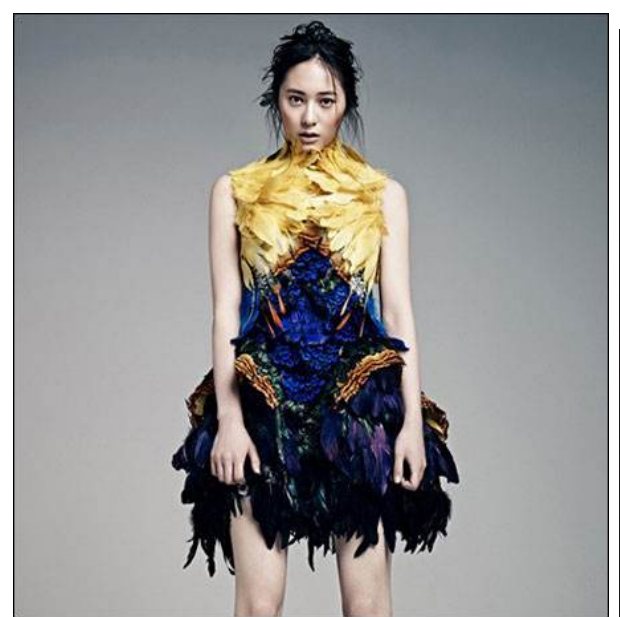

Fig.1 Costume Made from Feathers

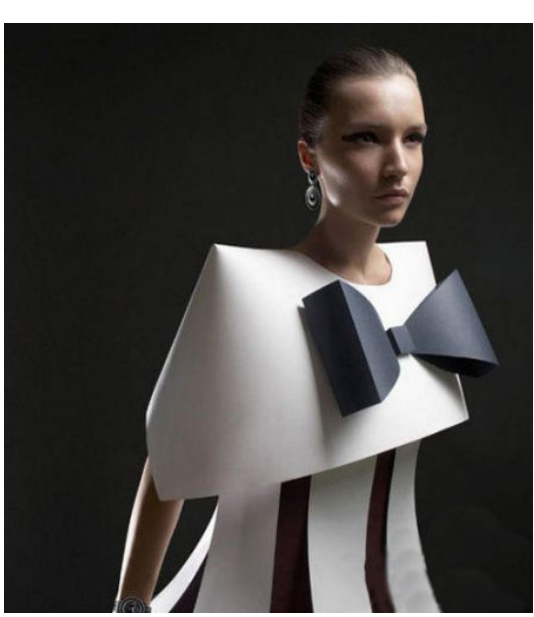

Fig. 2 Costume Made from Paper

\section{Color and pattern and style}

The existence of costume color must be attached to the costume fabric, which is an indispensable prerequisite for pattern and style. The combination of unique style, creative shape and vibrant, eyecatching color can better interpret the beautiful artistic conception.

\section{The need gratification role of costume color}

Vivid and beautiful costume color is not only dependent on the diversity of color, but also embodied in the personality of color. It is precisely because of people's continuous pursuit of the individuation difference of color that China has gradually changed its international costume color image of "blue, black and grey" since the reform and opening up. Nowadays, gorgeous clothes make up the beautiful scenery on the street, people have devoted infinite enthusiasm to costume color, and color has become the fashionable position for people to pursue the beauty of personality. The application of exaggerated and bold color contrast as well as exquisite color packing in costume design has always been regarded as a kind of great adventure, but what is more crucial is that, the ingenious designer can flexibly grasp the coordination between individuality of color and personality, and reasonably make use of such visual stimulation, thus exerting an unexpected effect and meeting the actual need of pursuing unique personality psychology.

In a word, color is an important means to express costume shape view, as well as an important carrier of costume emotions, so it is often regarded as the soul of clothing beauty. In the integration of costume and character image design, color exerts the largest and most direct impact on people. 
People can not only cognize color, but also have an instinctive demand for the harmony between colors.

\section{Choice and application methods of costume color in character image design}

\section{Costume color and accessories}

Accessories refer to costume ornaments, such as necklaces, earrings and rings in jewelry and hats, footwear and waistband. Accessories can play a complementary role in the overall effect of costume, but it is important to note that excessive accessories should be avoided in costume matching. The number of pieces of accessories in the appearance design of an overall character image should not exceed 5, thereinto, the number of pieces of ornaments cannot exceed 3, otherwise, a second-system effect will arise.

In the character image design, the overall grasp of the costume effect should be laid emphasis on, and the marvelous handling of accessory details can not be missed. The mutual complementation and coordination between the overall and the details are mainly embodied in the relation between reconciliation and contrast. Reconciliation refers to that on the premise of consistent style of costume with that of accessories, costume color and color of accessories should echo each other and be unified into a whole. Contrast means that the match of color, texture and pattern of costume with the color, material and style of accessories can produce a visual sense of beauty, such as the classical combination of black costume and silvery white diamond jewelry, and the texture contrast between rough fabric and delicate belt.

\section{Harmony and unity}

Each piece of clothing accessory can be viewed as a perfect artistic work. The matching beauty between clothing accessory and the clothing depends largely on the harmony and unity between the color, combination mode, texture and make-up effect of the accessory. Therefore, in costume matching, an uniform element in various forms should be detected out as far as possible. When the coat, base shirt, trousers and bag with different colors and made from different materials are matched together, the shoes in the same color with the coat can be chosen to generate a mutual echoing effect and to achieve unity. In addition, changes should be sought in the unified color or style. Only by combining the above two points tactfully, will top grade work with harmonious and aesthetic feeling be created.

\section{Contrast and reconciliation}

Contrast is very commonly used in the matching between costume color and accessories, which emphasizes on the highlight of opposite sides with the combination of opposite and different elements, so as to embody the exaggerated, intensive and distinct comparison between costume color and accessories. The reconciliation between costume color and accessories is divided into three types: first, similar structures, lines and shapes are regularly combined; second, costume and accessories with similar colors are matched; third, costume and accessories with similar textures, materials and hand feelings are organically combined to achieve a special visual effect.

\section{Rhythm and metre}

In the design of the matching between costume color and accessories, rhythm means the regular and orderly changes of combination factors in terms of light and heavy, strong and weak, big and small, curve and straight, more and less, and long and short. Rhythm can also refer to regular repetition within a certain range. Metre is a complex of intrinsic order and diversified changes.

\section{Costume color and age}

Regardless of the age group a wearer of costume belongs to, he or she can have unique charm and beauty. For a designer, only after the beauty of age as well as the real meaning and value of the beauty in different age groups was gotten, will the characteristics of costume color matching in various ages as well as the difference in the matching between the ages be deeply recognized, so that unparalleled good image can be designed according to the objects in different age groups.

Firstly, the meanings and values of completely different beauties in children, young people and middle-aged and old people should be learnt, so as to find out different contents and expressions of color in dressing. The meaning and value of beauty in children present as innocent, simple, curious 
and childish, in young people, they present as care-free, vigorous, enterprising, natural and energetic, in middle-aged people, they present as deliberate, subtle, quality-conscious and unique lingering charm, and in old people, they present as experienced, mentally mature and intelligent.

Secondly, from the perspective of the influence of external factors such as aesthetic tendency, fashion elements, social customs and age surroundings, the costume color matching in different age groups should not only adapt to the development and change of the corresponding beautyappreciation, but also adapt to the development and change of social beauty-appreciation. Children generally cannot make a choice on their own costume color, and their image-building of costume color is directly influenced by their parents' aesthetic idea. The costume color for children should be bright-colored-based but not vulgar, and be concise as far as possible. Youth is the season of life in which vigour can be fully reflected. Therefore, the costume color matching for young people has the widest range, the greatest potential and the most possibility to produce an effect, which can be lively and lucid or generous and solemn. For middle-aged people, their body type and temperament have changed greatly with age, so it is necessary to use costume color to shape a kind of overwhelming dignified and mature beauty. So costume for middle-aged people should adopt stable, gentle and simple but elegant tones, for example, dark color, middle tones and floral designs with different hues in gray tone are suitable. The most important thing for the design of costume for the elderly is to regain the confidence, so fashionable and fresh colors can be chosen, which does not make people feel uncomfortable, but instead foils the hale and hearty spirit of the elderly as well as the glow of health on their face, thereout, the elderly can surpass the young people, shape the image and build confidence through the costume.

\section{Costume color and style}

Character image design is a deep knowledge, which includes a complete set of theoretical system. "Color and style" are determined by two major factors. The relationship between the hair color and skin color of a person determines the overall coloring rule for the person, while the body line determines the rule of "shape" in dressing up, namely, the "personal style". As long as the "color and style" suitable for a person are found, the perfect overall image can be easily created.

The color in "color and style" includes costume color, cosmetic color, hairstyle color and accessory color. Costume color is the top priority among the overall colors, as well as the first impression of the overall image perception, it has extremely strong attraction, and can stress and highlight the particular style of characters. Cosmetic color can correct the skin color of humans and can enhance people's spiritual temperament. Hairstyle color and accessory color play the role of foil and embellishment, and can enhance the grade and taste of the overall image design. The style in "color and style" lays emphasis on the overall manner and style of people. Taking females as an example, there are many style types for them, such as maiden type, elegant type, romantic type and natural type, which requires that proper costume color should be selected based on the style positioning of character image.

\section{Conclusions}

Character image design is a comprehensive process, any link of which needs to take into account many factors, but the goal pursued is consistent from beginning to end, namely, to achieve "internal and external unity" of character image. The choice and application of costume color should not only start from the costume itself, but also take into full consideration age, style and other factors of the character objects, furthermore, the multiple needs of people and the society should be met through comprehensive and overlapping optimization design.

\section{References}

[1] Zhang Chen. Cultural charm capable of enhancing the corporate image [N]. Beijing Financial Daily, 2010-8-29.

[2] Jia Jingsheng. Costume color [M]. Beijing Higher Education Press, 2005. 